\title{
Atomic Imaging of Cracks
}

\author{
Using scanning transmission electron microscopy, researchers watch a 2D \\ crystal break apart one atomic bond at a time.
}

\author{
By Itamar Kolvin
}

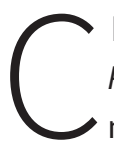

hemical elements are the star of Primo Levi's The

Periodic Table. In this collection of short stories-each named for a chemical element-the elements morph from serving as a literary device (for example, argon provides a metaphor for Levi's noble and inert ancestors) to acting as the central protagonist (at the end of the book, Levi recounts the odyssey of a carbon atom from lying in a mineral to residing in his own hand ). In a new study into the innermost workings of material fracture, an element-rhenium this time-also takes the lead and supporting roles [1]. Lingli Huang at the City University of Hong Kong and her colleagues successfully imaged the snapping of individual atomic bonds in a one-atom-thick sheet of rhenium disulfide $\left(\operatorname{ReS}_{2}\right)$ using scanning transmission electron microscopy. The demonstration was made possible because of the Re itself. Because of its unusual chemistry, Re forms a 2D lattice with "lanes" that guide cracks, allowing the cracks to propagate with ease [2]. The heavy element also efficiently deflects electrons, providing the signal needed to gain clear images. The study is a remarkable example of how a specific material can provide insight into the universal behavior of matter.
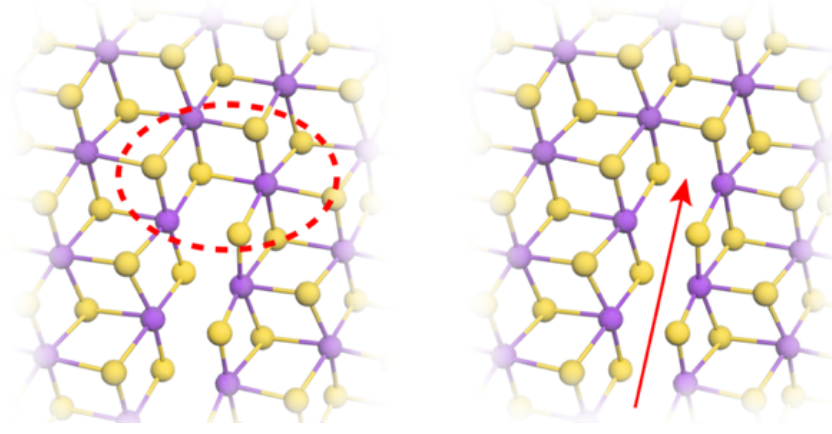

Figure 1: Researchers have directly observed the rupture of a two-dimensional crystal along its main crystallographic axis. Credit: APS/Joan Tycko
Breaking a material requires undoing the chemical bonds that hold it together. To do that, scientists need knowledge of the energies required to unravel the bonds between a material's atoms. But with that information in hand, predicting a material's resistance to fracture can still be surprisingly difficult-even if, like silicon, for example, the material breaks cleanly [3]. A hint of the origin of this complexity shows up in 2D materials, such as graphene and molybdenum disulfide. When they fracture, the 2D atomic lattices of these materials undergo a restructuring, which blunts cracks, frustrating their advance [4-6]. To help understand the sticking points in the theory, scientists need clear, atomic-resolution images of sharp, freely propagating cracks. Such images are exactly what the work of Huang and colleagues provides [1].

To make their measurements, the team used a technique called scanning transmission electron microscopy (STEM). Atoms are far too small to detect with visible light, but electrons, with their much smaller wavelengths-about 1/50th of an angstrom in this study-can easily resolve such features. In a STEM, a beam of electrons is accelerated in vacuum and then shaped by a series of capacitors and magnetic coils. This shaping process is similar to that of light by optical lenses. The electrons are focused to a $1-\AA ̊$ spot, which is then scanned across the sample. When the electrons hit an atom, they are scattered by the electrostatic potential of the atom's nucleus. The scattered electrons are collected and used to reconstruct an image of all the positions of the nuclei.

Because of its strong scattering, $\mathrm{ReS}_{2}$ provides an ideal target for STEM. (Interestingly, tungsten, the periodic-table neighbor of $\mathrm{Re}$, has just one fewer proton and is routinely used in electron microscopy to stain viruses and bacterial flagella.) The chemistry of Re also makes $\mathrm{ReS}_{2}$ a great material for studying fracture. $\operatorname{ReS}_{2}$ has a striped lattice structure, with the Re atoms 

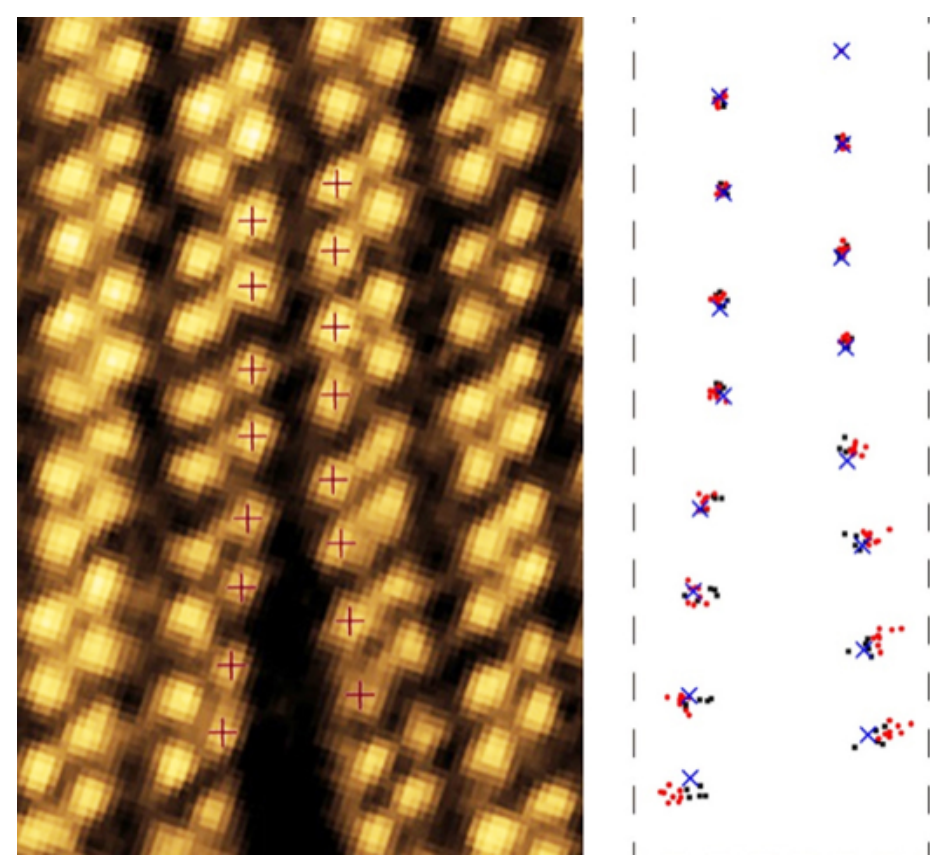

Figure 2: (Left) A planar STEM image of a propagating crack. The black plus symbols denote the predicted positions of the Re atoms. (Right) The measured positions of the Re atoms (black and red dots) along the crack match predictions (blue crosses).

Credit: L. Huang et al. [1]

arranged in rows that alternate in width (Fig. 1), providing easy paths for crack propagation.

In the experiments, Huang and colleagues placed a one-atom-thick sheet of $\mathrm{ReS}_{2}$ in the STEM. They induced multiple cracks in the sheet by blasting it with a high-intensity electron beam. They then reduced the beam intensity and scanned the beam across the sheet to monitor the evolution of the cracks. The team also captured images of healing events where bonds between broken Re atoms reformed.

The cracks formed between two Re stripes and then grew parallel to the stripes (Fig. 2). Careful scrutiny of the images revealed much more. By measuring the deformation of the lattice around the crack, the team showed that the tearing stresses were concentrated around the crack tip. The stresses then decayed as the inverse square root of the distance from the tip, a finding that matches predictions for macroscopic materials [7]. Using these measurements, the team defined a stress intensity threshold for cracks to propagate.

The team collected a new image every five seconds. Comparing consecutive images of a crack, they found that the crack progressed unevenly, with the number of newly broken bonds, $s$, in an image varying between zero and six. To ascertain the pattern of bond breaking, the team collected statistics of $s$. They found that the likelihood that the crack advanced by $s$ bonds between images decreased exponentially with increasing $s$. Such a pattern arises when there is a fixed probability $z$ for a bond to break in a given time period. The probability to break $s$ bonds between images is then proportional to $z^{\mathcal{S}}$, as the team found. In this experiment, they estimate that $z \sim 25 \%$, which agrees with a model that assumes that bonds break by thermal activation: a crack advances when the thermal motions of the atoms overcome the energetic barriers imposed by their bonds.

These results show the feasibility of studying crack propagation with STEM. As such, they are likely to inspire many others to make similar measurements on this and other materials. Some tweaks to the technique could make it even more effective. The images taken by Huang and colleagues used seconds-long exposure times, meaning they could only follow the propagation of the slowest crack (those that moved at a few angstroms per second). There is much interest in how faster cracks behave, as these cracks are subject to instabilities, meaning they can deviate from a straight line or form branches, for example [8,9]. To observe faster cracks, future experiments could use reduced exposure times. The intensity of the beam would then need to be increased to maintain sufficient contrast signal for imaging. Another future direction is studying bilayer systems where $\mathrm{ReS}_{2}$ might provide the forces necessary to drive cracks in an adjoining monolayer. But whatever path researchers take, there's certainly more to come from the fruitful combination of 2D materials, electron microscopy, and fracture mechanics.

Itamar Kolvin: Physics Department, University of California, Santa Barbara, CA, USA

\section{REFERENCES}

1. L. Huang et al., "In situ scanning transmission electron microscopy observations of fracture at the atomic scale," Phys. Rev. Lett. 125, 246102 (2020).

2. S. Tongay et al., "Monolayer behaviour in bulk $\mathrm{ReS}_{2}$ due to 
electronic and vibrational decoupling," Nat. Commun 5, 3252 (2014).

3. J. A. Hauch et al., "Dynamic fracture in single crystal silicon," Phys. Rev. Lett. 82, 3823 (1999).

4. K. Kim et al., "Ripping graphene: Preferred directions," Nano Lett. 12, 293 (2011).

5. T. Hue Ly et al., "Dynamical observations on the crack tip zone and stress corrosion of two-dimensional $\mathrm{MoS}_{2}$," Nat. Commun. 8, 14116 (2017).

6. S. Wang et al., "Atomically sharp crack tips in monolayer $\mathrm{MoS}_{2}$ and their enhanced toughness by vacancy defects," ACS Nano 10, 9831 (2016).

7. G. R. Irwin, "Analysis of stresses and strains near the end of a crack transversing a plate.," Trans. ASME, Ser. E, J. Appl. Mech. 24, 361 (1957).

8. E. Bouchbinder et al., "The dynamics of rapid fracture: Instabilities, nonlinearities and length scales," Rep. Prog. Phys. 77,046501 (2014).

9. J. R. Kermode et al., "Macroscopic scattering of cracks initiated at single impurity atoms," Nat. Commun. 4, 2441 (2013). 